\title{
Glucocorticoid-Induced Hypermetabolism in White Adipose Tissue in Cushing Syndrome
}

\author{
Sasha O. Staack ${ }^{1}$, Allison C. Rosenthal ${ }^{2}$, Curtiss B. Cook $^{3}$, and Ming Yang ${ }^{1}$ \\ ${ }^{1}$ Department of Radiology, Mayo Clinic, Scottsdale, Arizona; ${ }^{2}$ Department of Hematology/Oncology, Mayo Clinic, Scottsdale, \\ Arizona; and ${ }^{3}$ Division of Endocrinology, Mayo Clinic, Scottsdale, Arizona
}

\begin{abstract}
An adult lymphoma patient developed Cushing syndrome after short-term, high-dose dexamethasone administration and presented with a distinctive Cushingoid fat redistribution pattern and associated increased ${ }^{18} \mathrm{~F}-\mathrm{FDG}$ uptake in white adipose tissue. Recognition of the unique ${ }^{18} \mathrm{~F}-\mathrm{FDG}$ uptake manifestation may aid in the diagnosis of this iatrogenic syndrome and avoid image misinterpretation.
\end{abstract}

Key Words: Cushing syndrome; white fat; glucocorticoids; PET/CT; FDG

J Nucl Med Technol 2020; 48:285-286

DOI: 10.2967/jnmt.119.237545

\section{$\mathbf{I}_{\mathrm{n}}$} addition to regulation of glucose metabolism, glucocorticoids play an important role in adipose metabolism via multiple pathways. Exogenous high-dose glucocorticoids may cause Cushing syndrome, with the resultant classic redistribution of white adipose tissue. Although uncommon, the redeposited adipose tissue might show enhanced ${ }^{18} \mathrm{~F}$ FDG uptake under certain clinical conditions and lead to misinterpretation.

\section{CASE REPORT}

A 59-y-old woman with a history of renal transplantation developed a posttransplant lymphoproliferative disorder involving the skin and brain, which was identified on ${ }^{18} \mathrm{~F}-\mathrm{FDG}$ PET/CT scans. She was treated with rituximab for chemotherapy and dexamethasone, $8 \mathrm{mg} / \mathrm{d}$, for controlling cerebral edema. She also had been on low-dose prednisone, $5 \mathrm{mg} / \mathrm{d}$, for immunosuppression after her renal transplantation. Six weeks after initiation of chemotherapy and combined glucocorticoid therapy, a follow-up ${ }^{18}$ F-FDG PET/CT scan was performed with a fasting blood glucose level of $110 \mathrm{mg} / \mathrm{dL}$. On ${ }^{18}$ F-FDG PET/CT images, both the brain and the cutaneous lesions had resolved. However, there was rapid proliferation of subcutaneous white adipose tissue with increased

Received Oct. 1, 2019; revision accepted Nov. 21, 2019.

For correspondence or reprints contact: Ming Yang, Department of Radiology, Mayo Clinic Arizona, 13400 E. Shea Blvd., Scottsdale, AZ 85259.

E-mail: yang.ming@mayo.edu

Published online Dec. 6, 2019.

COPYRIGHT (C) 2020 by the Society of Nuclear Medicine and Molecular Imaging.
${ }^{18}$ F-FDG uptake at the face, neck, and back (Fig. 1). Subcutaneous white fat at the posterior upper neck thickened from 2.1 to $5.1 \mathrm{~cm}$, with $\mathrm{SUV}_{\max }$ increasing from 1.2 to 2.6. In both thighs, there was increased ${ }^{18}$ F-FDG uptake by intermuscular adipose tissue beneath the fascia of wasting muscles, with $\mathrm{SUV}_{\max }$ increasing from 1.0 to 3.6 (Fig. 2). Because there were no corresponding lesions in the redistributed fatty tissue, the hypermetabolism was considered a treatment-related false-positive finding, and a diagnosis of iatrogenic Cushing syndrome was achieved. A dexamethasonetapering protocol ensued, given the favorable response to therapy.

\section{DISCUSSION}

Enhanced ${ }^{18}$ F-FDG uptake in remodeled white adipose tissue has been sparsely reported in lymphoma patients

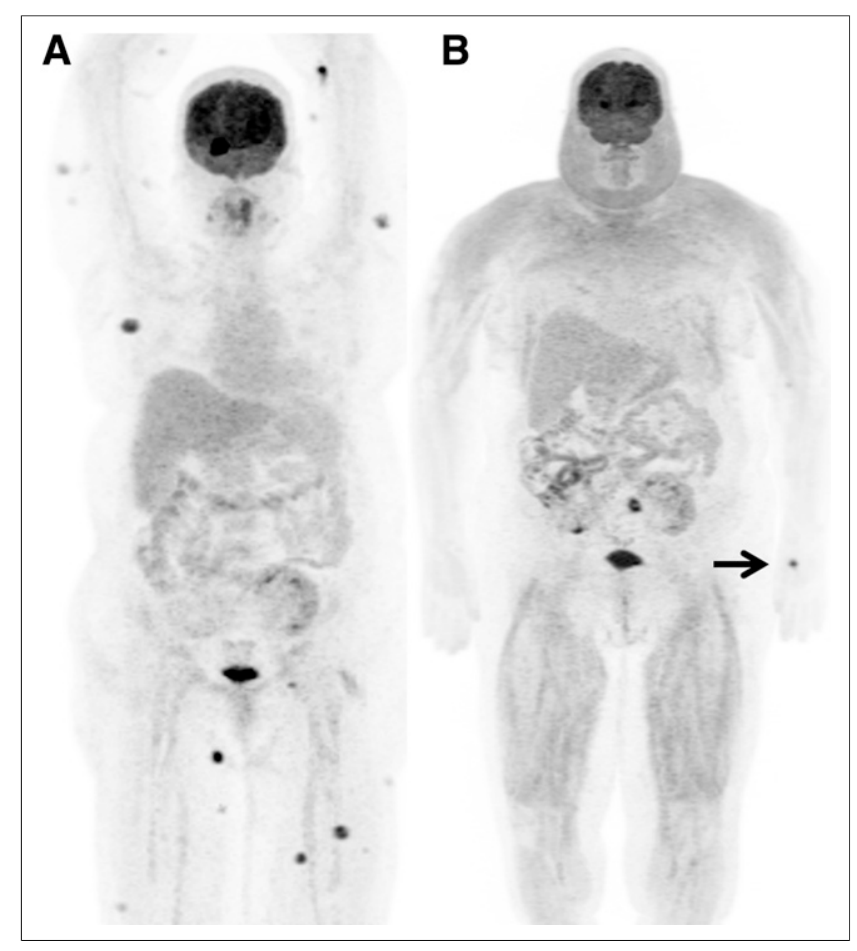

FIGURE 1. Maximum-intensity projections of baseline $(A)$ and follow-up (B) ${ }^{18} \mathrm{~F}-\mathrm{FDG}$ PET/CT scans demonstrate resolution of numerous cutaneous and right-temporal-lobe tracer-avid lesions (arrow in B indicates ${ }^{18} \mathrm{~F}-\mathrm{FDG}$ injection site). 


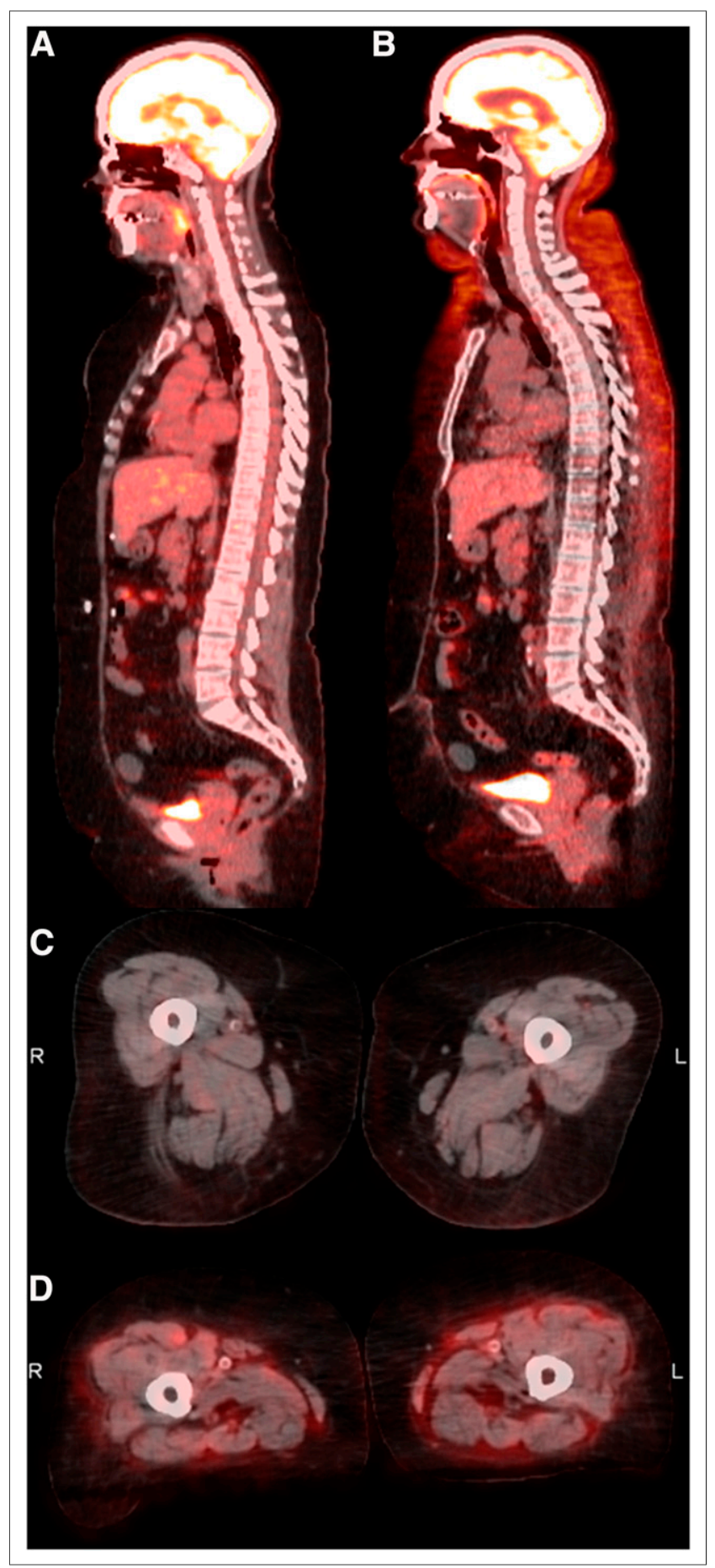

FIGURE 2. Sagittal fused images at baseline (A) and follow-up (B) show thickened subcutaneous white adipose tissue with increased ${ }^{18} \mathrm{~F}-\mathrm{FDG}$ uptake at face, neck, and back. Axial fused images of thighs at baseline (C) and follow-up (D) reveal bilateral muscle wasting and hypermetabolic intermuscular adipose tissue. receiving chemotherapy and glucocorticoids $(1,2)$. It is well known that glucocorticoids play an important role in regulation of fat metabolism via stimulation of lipolysis, lipogenesis, and adipogenesis processes, which may lead to a classic Cushingoid distribution of central white adipose tissue, that is, moon face and buffalo back, etc. The increased ${ }^{18} \mathrm{~F}$-FDG uptake in white adipose tissue might be associated with upregulated glycolytic mitochondrial metabolism and proinflammatory macrophage recruitment caused by dexamethasone administration (3). It has also been found that glucocorticoids promote differentiation of preadipocytes into mature adipocytes, which have relatively high glucose transporter 1 (GLUT 1) expression (4).

Both thighs showed intermuscular adipose tissue with increased ${ }^{18} \mathrm{~F}$-FDG uptake. The lower-extremity intermuscular adipose tissue is considered an ectopic fat depot similar to visceral fat, which is part of the central white adipose tissue family (5). Therefore, we believe that the hypermetabolism of intermuscular adipose tissue might be due to the same mechanism as that for central subcutaneous white fat tissue.

\section{CONCLUSION}

Enhanced ${ }^{18} \mathrm{~F}-\mathrm{FDG}$ uptake in redistributed white adipose tissue in glucocorticoid-induced Cushing syndrome is a multifactorial phenomenon and might be related to such factors as age, sex, and dosage and duration of glucocorticoid exposure. Recognition of the uncommon ${ }^{18}$ F-FDG uptake pattern in hypertrophic central white adipose tissue may raise awareness of iatrogenic Cushing syndrome and avoid misinterpretation.

\section{DISCLOSURE}

No potential conflict of interest relevant to this article was reported.

\section{REFERENCES}

1. Hwang DY, Lee JW, Lee SM, Kim S. Causes of ${ }^{18}$ F-FDG uptake on white adipose tissue. Hell J Nucl Med. 2016;19:7-9.

2. Kong MC, Nadel HR. ${ }^{18}$ F-FDG PET/CT with diffusely high FDG uptake throughout subcutaneous adipose tissues. Clin Nucl Med. 2018;43:762763.

3. Pattison DA, Hofman MS, Lau E, Ware R, Hicks RJ. Enhanced white adipose tissue metabolism in iatrogenic Cushing's syndrome with FDG PET/CT. J Clin Endocrinol Metab. 2014;99:3041-3042.

4. Gould GW, Holman GD. The glucose transporter family: structure, function and tissue-specific expression. Biochem J. 1993;295:329-341.

5. Addison O, Marcus RL, Lastayo PC, Ryan AS. Intermuscular fat: a review of the consequences and causes. Int J Endocrinol. 2014;2014:309570. 